\title{
Outcomes of multidisciplinary team interventions in the management of sickle cell disease patients with opioid use disorders
}

\author{
$A$ retrospective cohort study
}

Omar Al Zahrani, MD, Ehab Hanafy, MD, Osama Mukhtar, MSc, Ashraf Sanad, MD, Wael Yassin, MD.

\begin{abstract}

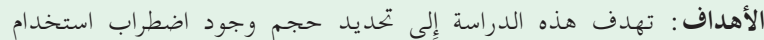

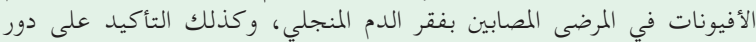

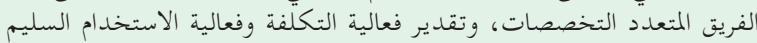

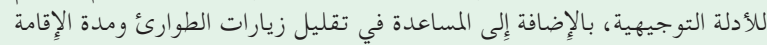

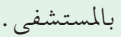

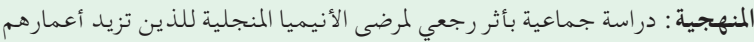

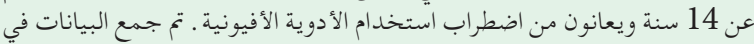

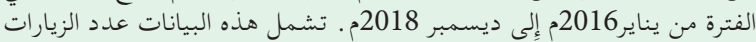

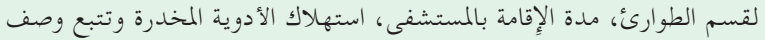

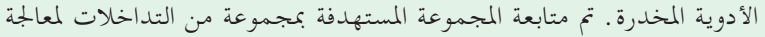
الألم واستخدام موارد الرعاية الصحية.

النتائج: تم تحديد 21 مريضا يعانون من اضطراب استخدام الأفيونات، و و بعد

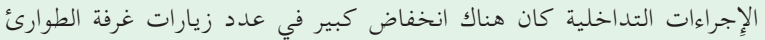

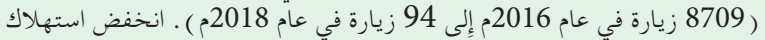

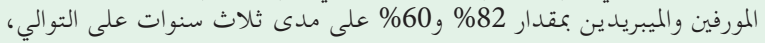

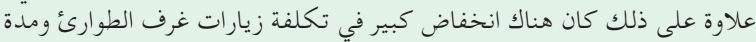

الإقامةبالمستشفى على ذاوك كان

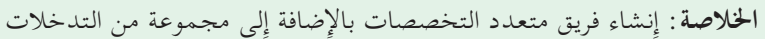

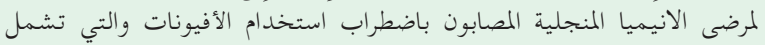

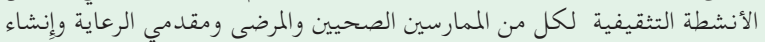

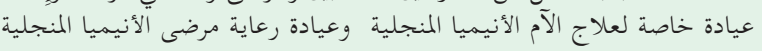

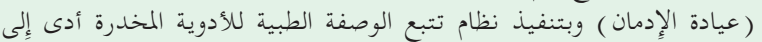

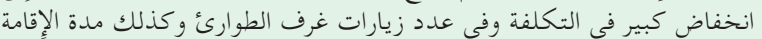

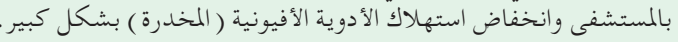

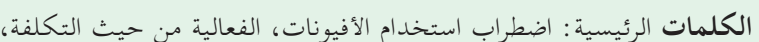

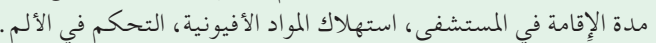

Objectives: To identify the magnitude of opioid use disorder (OUD) among sickle cell disease (SCD) patients; emphasize on multidisciplinary team (MDT) role; estimate cost-effectiveness following the proper use of therapeutic guidelines; and facilitate the reduction of emergency room (ER) visits and the length of stay (LOS).

Methods: This retrospective cohort study included SCD patients aged 14 years and above, who have OUD. Data was collected between January 2016 and December 2018. Data included ER visits, hospital LOS, opioid consumption, and narcotic prescription tracking. The target group was followed with a set of interventions for pain management and healthcare resource utilization.

Results: Twenty one SCD patients were identified with OUD. Following the interventions, there was a statistically significant decrease in ER visits of these OUD patients (from 8709 visits in 2016 to only 94 in 2018). Morphine consumption decreased by $82 \%$ and meperidine by $60 \%$, over the 3 -year period. Moreover, there was a huge reduction in both ER and LOS costs for this cohort of patients.

Conclusion: Establishing an MDT and a series of interventions for SCD patients with OUD, including educational activities for caregivers and patients; establishing a palliative/pain clinic and a SCD addiction clinic; and implementing an adequate opioid prescription tracking system resulted in a significant reduction in both the cost and number of ER visits and hospital LOS and dramatically decreased opioid consumption.

Keywords: opioid use disorder, cost-effectiveness, length of stay, opioid consumption, pain management

$$
\begin{array}{r}
\text { Saudi Med J 2020; Vol. } 41 \text { (10): 1104-1110 } \\
\text { doi: 10.15537/smj.2020.10.25386 }
\end{array}
$$

From Prince Sultan Oncology Center (Al Zahrani, Hanafy, Mukhtar. Yassin), and from the Department of Psychiatry (Sanad), King Salman North West Armed Forces Hospital, Tabuk, Kingdom of Saudi Arabia.

Received 4th June 2020. Accepted 1st September 2020.

Address correspondence and reprint request to: Dr. Omar Al Zahrani, Prince Sultan Oncology Center, King Salman North West Armed Forces Hospital, Tabuk, Kingdom of Saudi Arabia. E-mail: psoc.research@gmail.com

ORCID ID: https://orcid.org/0000-0001-7344-1462 
Sickle cell disease (SCD) is a common autosomal $\checkmark$ recessive hematologic disorder in the Kingdom of Saudi Arabia. Prevalence of sickle cell trait ranges from $2-27 \%$. The disease is characterized by the production of abnormal readily polymerized hemoglobin $S$ that is responsible for the characteristic disease complications. ${ }^{1}$ Vaso-occlusive crisis (VOC) is the most common among SCD complication and is responsible for most emergency room (ER) visits and hospital admissions. ${ }^{2}$ Analgesics are the cornerstone for VOC treatment with varying doses and types according to the severity of pain. Han et al, ${ }^{3}$ reported that opioid medications were used by $39.9 \%$ of patients, and that only $31.8 \%$ of patients took immediate-release and $0.4 \%$ of patients took extended-release opioids, while $7.8 \%$ have used both forms. Patients with avascular necrosis were found to use higher doses. The rate of opioid use increases dramatically with age peaking at $58.3 \%$ in patients between 20-29 years of age. ${ }^{3}$ Numerical pain scores are extensively used in pain assessment for SCD patients; however, it can be misleading, especially in a group of patients with a high risk of developing opioid use disorder (OUD). ${ }^{4}$ Therefore, a more objective method of pain assessment and re-assessment is essential for proper management and avoiding unnecessary interventions. There is a limited number of studies that describe OUD in patients with SCD. In this study, we identified a group of SCD patients with OUD who showed a significant decrease in dependence on hospital resources (including medication, hospital stays, and ER visits) after successful interventions adopted by the King Salman North West Armed Forces Hospital (KSAFH), Tabuk, Saudi Arabia.

Methods. This retrospective cohort study of SCD patients with OUD was carried out in KSAFH, Tabuk, Saudi Arabia which serves as a tertiary care hospital and referral center for the northwestern region of the country. An ethical approval for the study was obtained from the Ethical and Research Committee of the hospital.

The inclusion criteria were as follows: SCD patients, aged $>14$ years and who have confirmed diagnosis of OUD were included in this study. The exclusion criteria were as follows: SCD patients aged 14 years or less; SCD patients ( $>14$ years) without OUD.

Disclosure. Authors have no conflict of interests, and the work was not supported or funded by any drug company.
In the view of the problems like frequent ER visits, increased length of stay, reiterated aggression incidences by a group of SCD patients, a multidisciplinary team (MDT) was formed and have set some investigations to establish the diagnosis of patients with SCD who have OUD. These included the justification for ER visits and long hospital stays with the use of detailed medical reports and review of medical files. Moreover, to confirm the diagnosis of OUD, the assessment by a psychiatrist with addiction specialty is mandatory.

After defining the magnitude of the problem, the targeted group was subsequently followed with a set of interventions for optimum pain management and health care resource utilization. The following pathway elaborates set of interventions and working mechanism of the MDT (Figure 1).

The data regarding number of ER visits, hospital length of stay (LOS), amount of opioid consumption in

Super hospital utilization (frequent ER visits, increased length of

2. Verbal and physical aggression by a group of patients with $\mathrm{SCD}$ 3. Repeated OVA incidents for this group of patients
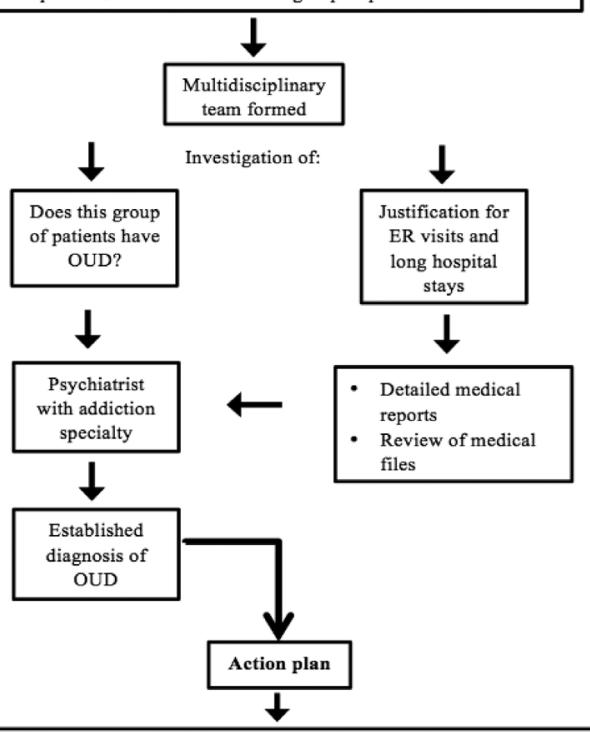

1. Assessment of the magnitude of the problem by biostatisticians and hospital administration

2. Awareness and education about the problem for related medical staff

3. Awareness and education for the patients/families via individual and group meetings

4. Establish a pain and palliative care clinic

5. Establish an SCD care clinic run by a psychiatrist (for diagnosis and management)

6. Track and monitor opioid prescriptions in the ER and hospital admission wards

7. Regular MDT meetings to follow up the progress

Figure 1 - The set of interventions and working mechanism of the multidisciplinary team (MDT). ER: emergency room, SCD: sickle cell disease, OUD: opioid use disorder, OVA: occurrence, variance, and accident 
the ER and in-patient wards, and narcotic prescription tracking were collected for SCD patients who have OUD under regular follow up for a 3-year period between January 2016 and December 2018.

Statistical analysis. All demographic and clinical data were collected and analyzed using the Statistical Package for Social Sciences, version 20 (IBM Corp, Armonk, NY, USA). Frequencies and percentages of the descriptive analysis of the data were presented in tabulated and graphical form. Paired sample t-test was used to compare the ER visits of this cohort of patients during the different years of the study period (2016 to 2018). A $p$-value $<0.05$ was considered significant.

Results. After a thorough investigation, 21 SCD patients were reported as diagnosed with OUD. Eighteen (67\%) of these were male and majority (14, $67 \%$ ) were aged 26 to 35 years. The frequency of the ER visits was gradually decreased during the successive months of the study period (Figure 2). Paired sample t-test was used to compare the number of ER visits of this cohort of OUD patients for 3 consecutive years (2016 to 2018) and there was a statistically significant decrease in number of ER visits from 8709 in 2016, to 799 in 2017, to 94 in 2018 ( $p=0.00)$ (Tables $1 \& 2$ ). In addition, there was a dramatic reduction in the cumulative hospital LOS of these SCD patients with OUD from 2051 days in 2016 to 80 days in 2018
(Table 2).

Tracking and monitoring of the opioid prescriptions in the emergency department and hospital admission wards was one of the most important task in the action plans of the MDT. As a results of the interventions, there was a significant reduction in the hospital use of parenteral morphine $(10 \mathrm{mg} / \mathrm{ml})$ in both ER visits (11940 ampules in 2016 vs. 2088 ampules in 2018) and in-patient (29149 ampules in 2016 vs. 11412 ampules in 2018) admissions (Table 3). There was also a significant reduction in the hospital consumption of parenteral meperidine over the study period.

Table 1 - Statistical analysis between emergency room visits in 2016 to 2018 for SCD patients (cohort of 21 patients) with OUD.

\begin{tabular}{|c|c|c|c|c|}
\hline Comparison & Mean \pm SD & T-statistics & $P$-value & Result \\
\hline ER visits in 2016 & $414.7 \pm 227.8$ & & & Average ER \\
\hline ER visits in 2017 & $38.05 \pm 49.1$ & 8.24 & 0.00 & $\begin{array}{l}\text { visits in } 2017 \text { are } \\
\text { significantly lower } \\
\text { than in } 2016\end{array}$ \\
\hline ER visits in 2017 & $38.05 \pm 49.1$ & & & Average ER \\
\hline ER visits in 2018 & $4.48 \pm 5.9$ & 3.21 & 0.00 & $\begin{array}{c}\text { visits in } 2018 \text { are } \\
\text { significantly lower } \\
\text { than in } 2017\end{array}$ \\
\hline ER visits in 2016 & $414.7 \pm 227.8$ & & & Average ER \\
\hline ER visits in 2018 & $4.48 \pm 5.9$ & 8.29 & 0.00 & $\begin{array}{c}\text { visits in } 2018 \text { are } \\
\text { significantly lower } \\
\text { than in } 2016\end{array}$ \\
\hline
\end{tabular}

ER: emergency room, SCD: sickle cell disease, OUD: opioid use disorder

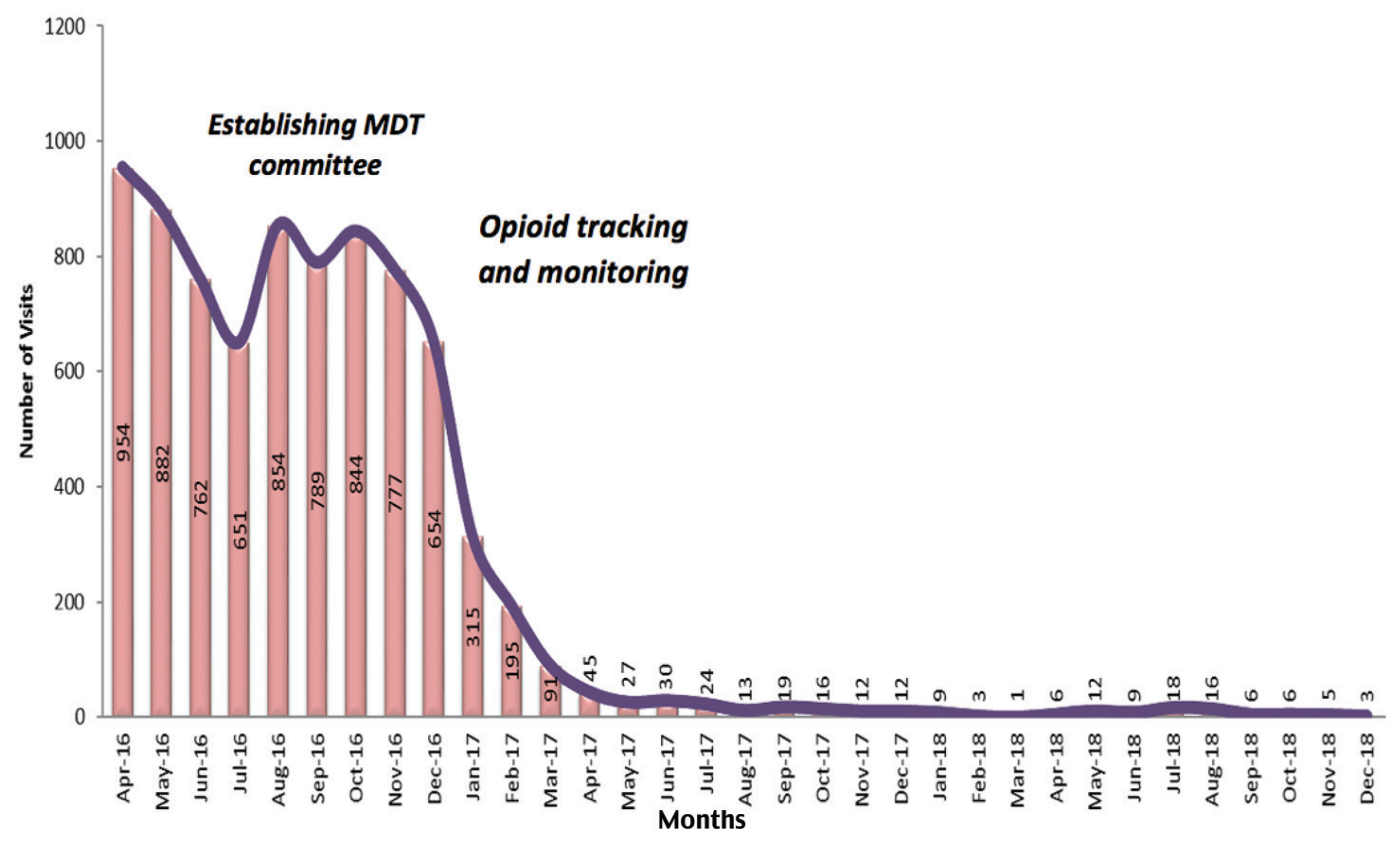

Figure 2 - Cumulative number of emergency room visits for patients with sickle cell disease comorbid with opioid use disorder (Cohort of 21 Patients) from April 2016 to December 2018. MDT: multidisciplinary team 
Table 2 - Comparison of accumulated ER and admissions costs endured by the hospital in 2016 to 2018 for SCD patients (cohort of 21 patients) with OUD.

\begin{tabular}{cccrc}
\hline Year & $\begin{array}{c}\text { Number of } \\
\text { ER visits }\end{array}$ & $\begin{array}{c}\text { ER visits } \\
\text { Cost endured by } \\
\text { the hospital (USD) }\end{array}$ & LOS & $\begin{array}{c}\text { Admissions } \\
\text { Cost endured by the } \\
\text { hospital (USD) }\end{array}$ \\
\hline 2016 & 8709 & 7141380 & 2051 & 6173510 \\
2017 & 799 & 655180 & 256 & 770560 \\
2018 & 94 & 77080 & 80 & 240800 \\
\hline
\end{tabular}

ER: emergency room, SCD: sickle cell disease, OUD: opioid use disorder, USD: American dollars, LOS: length of stay

Table 3 - Consumption rate of parenteral morphine $(10 \mathrm{mg} / \mathrm{ml})$ and meperidine (2016 to 2018).

\begin{tabular}{lcccc}
\hline \multicolumn{2}{c}{ Morphine } & \multicolumn{2}{c}{ Meperidine } \\
Year & ER & In-patient & ER & In-patient \\
\hline 2016 & 11940 & 29149 & 9194 & 27439 \\
2017 & 3159 & 10848 & 3336 & 10801 \\
2018 & 2088 & 11412 & 334 & 8665 \\
\hline \multicolumn{3}{c}{ ER: emergency room } \\
\hline
\end{tabular}

Consequently, at the end of all these implementations furnished by MDT for 3 consecutive years, we were in a position to compare the cost endured by the hospital in the services for this cohort of OUD patients. The cost borne by the hospital for ER visits by this cohort of patients decreased by $99 \%$ (Table 2). The total cost of admissions decreased by $96 \%$ (Table 3). The cost of services was calculated according to a study in 2009 by Ballas ${ }^{5}$ and according to hospital costs estimated by the World Health Organization (WHO) in KSA. ${ }^{6}$

Discussion. Pain is a hallmark comorbid symptom of SCD and accounts for the majority of pediatric and adult admissions and ER visits. The pain characteristics are unique, as the pain is unpredictable and occurs frequently due to repeated vaso-occlusive and painful crises. In addition, chronic pain, which manifests more in adults, can lead to poor quality of life. ${ }^{7}$

Pain can be a significant feature of SCD for patients who lack effective treatment, particularly diseasemodifying agents such as hydroxyurea, and who inappropriately use analgesics. A proportion of SCD patients known as super hospital utilizers are responsible for a disproportionate number of ER visits and hospital admissions, defined as $\geq 12$ admissions per year. ${ }^{8}$

A retrospective cohort study was carried out to identifying a group of super utilizer SCD patients diagnosed with OUD. We compared the rate of hospital utilization, opioid consumption, ER visits, and hospital admissions in addition to cost-effectiveness before and after implementing an intensive interventional strategy to solve this problem.

Twenty-one patients (mostly male) representing 9\% of adult patients with SCD were diagnosed with OUD. Some evidence suggests that the prevalence of OUD among SCD patients is similar to general United States (US) population. ${ }^{9}$ Martin et $\mathrm{al}^{10}$ reported an incidence of OUD ranging from $0.2 \%$ to $2 \%$ in SCD patients in the US. Higher incidences were reported in different parts of Nigeria $(17.8 \%$ in the northeast and $10 \%$ in the southeast) with a higher proportion of males. ${ }^{11,12}$ These values confirm the incidence variation according to geographical location; however, the higher incidence in our study can be attributed mainly to the clear deviation from the clinical practice guidelines.

Opioid use disorder at our hospital was noticed as a result of super hospital utilization in the form of frequent ER visits, increased LOS, aggressive behavior on the part of patients with SCD, and an increased number of incidents involving this cohort of patients, as detected by the occurrence, variance, and accident (OVA) incident reporting system. After interviewing these patients, it was found that several factors contributed to the incidents, including chronic pain syndrome and psychosocial issues, such as financial problems and depression. Interestingly, Jonassaint et $\mathrm{al}^{13}$ found a significant overlap between depression and chronic pain in patients with SCD, where patients with depression had almost 3 times greater relative risk of being high health care utilizers and 50\% higher rate of hospitalization (2.9 vs. 1.8 hospitalizations per year).

As an initial step, an MDT was formed (Table 4) to determine whether these patients have OUD or not and to examine whether the frequent ER visits and LOS were justifiable. Subsequently, a consultant psychiatrist and addiction specialist succeeded in proving the diagnosis of these patients using the DSM-5 diagnostic criteria. ${ }^{14}$ A thorough investigation of patients' medical records and a review of complete medical reports obtained from the responsible physicians confirmed a clear diagnosis

Table 4 - List of multidisciplinary team members.

\begin{tabular}{lc}
\hline Main MDT members & Supporting team \\
\hline Consultant hematologist & Health educator \\
Internal medicine representative & Social worker \\
Pain and palliative care service consultant & Religious affairs representative \\
Consultant psychiatrist & Quality representative \\
Pharmacist & Biostatistician \\
Nursing staff representative & Secretary \\
Emergency department supervisor & \\
\hline \multicolumn{2}{c}{ MDT - multidisciplinary team. } \\
\hline
\end{tabular}


of OUD for this cohort of patients. At this point, an intensive interventional strategy was initiated.

A limited number of studies in the literature have highlighted OUD in patients with SCD, and some authors have clarified the effectiveness of implementing different types of interventions to reduce the rate of ER visits and hospital stays. In their retrospective cohort study, Koch et al, ${ }^{15}$ compared the rate of admission before and after the opening of a clinic for adults with SCD. They found that a management strategy at the clinic focusing on super-utilizing adults with SCD lowered the admission rate.

Other previous study has focused on the hospital days for patients with uncomplicated painful crises and on a comprehensive model of care and its impact on morbidity and mortality. However, none have been concerned with SCD patients with a diagnosis of OUD. ${ }^{16-18}$

This study is focus on OUD in patients with SCD and to emphasize the need to establish an intensive interventional strategy to improve this problem. This strategy (action plan) includes 7 main points. The first step is to improve the awareness and education of medical staff. Patients with SCD and VOC can be either undertreated or overtreated. Physicians and nurses have been known to have negative attitudes on pain management for patients with SCD. In a survey by Shapiro et $\mathrm{al},{ }^{19}$ stated that $53 \%$ of ER physicians and $23 \%$ of hematologists think that more than $20 \%$ of adult patients with SCD have OUD. Another study showed that $63 \%$ of nurses believed that OUD is common among adult SCD patients, and 30\% are reluctant to give the recommended dose of opioids upon evaluation. ${ }^{20}$ The consequence of this systematic bias is the undertreatment of pain for many adults with SCD.

Following guidelines and sticking to policies are mandatory to treat patients in an evidence-based approach. We achieved awareness and education for the staff through rounds, regular meetings with medical staff, workshops, and presentations. Furthermore, updated guidelines were distributed in different hospital departments to improve physicians' knowledge regarding the standard of care for patients with SCD, particularly those experiencing a painful crisis. The importance of awareness of SCD has been addressed in many studies. For instance, one study suggests that the attitude of clinicians toward SCD patients may be improved through a short and relatively easy to implement intervention. That study used an 8-minute video as an educational resource for health care providers addressing the challenges of pain management and the patient perspective in managing acute painful crises. ${ }^{21}$

Similarly, there has been much attention focused on awareness and education for patients and their caregivers. For example, individualized and group meetings have been very successful in identifying different factors that contribute to high hospital utilization. Addressing these factors (namely, social issues and financial problems) and making appropriate referrals to specialists (namely, social workers, health educators, and SCD psychiatric clinics) have helped significantly in managing patients with OUD.

An important topic outlined in educational programs is pain assessment tools. Numerical pain scales as a tool for measuring the degree of pain have been widely used. For example, this method has been used by the American Pain Society since 1996 (pain as the 5th vital sign). Twenty years later, many researchers have found that these scaling tools directly contributed to America's opioid prescription epidemic, and efforts shifted towards developing a more objective assessment tool for pain severity. ${ }^{22,23}$ One tool is the functional activity scale (FAS), which measures the degree of activity limitation ("no" or "mild" or "severe" limitations) as a consequence of uncontrolled pain..$^{24,25}$ During our study, we realized the inadequacy of numerical pain scales vis-à-vis opioid use, particularly for the super hospital utilizers, and started implementing a more objective, multidimensional pain scale that integrates functional activity and behavioral attitudes to pain. Some elements are the general appearance of the patient (namely, posture, facial expression, gait, mobility), sleep characteristics, behavior towards medical staff, and the pattern and quality of pain. Despite not being validated yet, this pain assessment tool has proven to be effective in determining the severity of pain and has helped significantly in subsequent management plans.

Establishing specialized clinics for SCD, including a pain/palliative care clinic and a SCD care clinic, was the transformation point in the ideal management of SCD patients with OUD. The main goal of the pain and palliative care clinic is to address all the dimensions of pain and to induce the highest possible functional capacity, taking into consideration the psychosocial and physical aspects of the patients. It is concerned with the proper assessment of pain using different tools, strict adherence to clinical guidelines, and the appropriate referral of patients when indicated. The clinic is responsible for closely following up and monitoring ER visits, admissions, and the opioid consumption of SCD patients with OUD who were not known to have regular follow ups at ordinary SCD clinics. The SCD care clinic (psychiatric clinic) has vital roles that include 
the following: i) providing supportive psychotherapy to SCD patients and their caregivers; ii) improving the quality of life of SCD patients; 3) improving SCD patients' coping mechanisms to deal with the disease itself (including its various complications) and with pain (through patient education, cognitive therapy, behavioral therapy, and psychodynamic psychotherapy); iv) ensuring early diagnosis and proper management of any psychiatric disorders in SCD patients; v) ensuring the awareness of SCD patients not suffering from OUD on the potential problem of OUD (primary prevention); vi) ensuring the early detection of warning signs of OUD in SCD patients; vii) diagnosing SCD patients who are suffering from OUD; viii) providing a detoxification program to SCD patients suffering from OUD; ix) detoxification includes 3 steps: a) evaluating patients to identify the opioids and amounts being used, assessing potential comorbid disorders, making dual diagnoses, and identifying behavioral or mental issues; b) stabilizing patients during the process of detoxification [mostly via pharmacotherapy], explaining to patients what to expect during treatment and recovery, and involving close caregivers to show support; c) guiding patients through the detoxification process to prepare them for the recovery phase, which includes motivating patients to complete the process by joining a rehabilitation program; $x$ ) supervising SCD patients suffering from OUD during rehabilitation programs at other health facilities; xi) following up SCD patients who suffered from OUD after rehabilitation programs to prevent relapses.

In addition to the above interventions, we largely attribute our successful management results to opioid prescription tracking and monitoring without compromising patient care. A qualitative study showed that restricting opioids may have negatively affected patients' care. Patients reported decreased opioid dosing, increased stigmatization regarding opioid use, physician preoccupation with opioid dosage interfering with comprehensive care, and lack of access to alternative therapies. This study concluded that patients with SCD should be included in establishing goals for managing pain and improving functionality using multimodality approaches. ${ }^{26}$ Subsequently, we tried to engage the patients in decision making and management planning.

The process of tracking and monitoring opioid use was very strict, starting with prescribing the opioid analgesics through to the patients receiving them. The process included daily reports regarding SCD patients who visited the ER or who were admitted, including the reasons for visits or admissions; LOS; management plans and type and dose of analgesics received; and whether there were deviations from the guidelines. All the reports were received, reviewed, and interpreted based on the standard guidelines by the MDT, which met on a regular basis.

Study limitations. Being retrospective is the main limitation of this study. In addition, this was a single hospital-based study that is subjected to selection bias, recommendations cannot be generalized due to this fact and due to small sample size. A prospective, multicenter study needs to be initiated in order to establish some more standard recommendations.

In conclusion, this intensive intervention strategy could not have been established without a dedicated MDT consisting of highly experienced health care providers. It is well known that multidisciplinary care for SCD patients provided in the context of a chronic care model can result in significant improvements in important quality targets and may reduce acute resource use. ${ }^{27}$

Various factors contributed to the success of the MDT, including ease of patient access to the team, improved service coordination, the expedition of referrals, and direct supervision and authority. The most important benefits were treatment recommendations based on clinical information and multidisciplinary assessment, adherence to guidelines, increased team competence, and increased patient safety.

\section{References}

1. Jastaniah W. Epidemiology of sickle cell disease in Saudi Arabia. Ann Saudi Med 2011; 31: 289-293.

2. Lanzkron S, Carroll CP, Haywood C Jr. The burden of emergency department use for sickle-cell disease: an analysis of the national emergency department sample database. Am J Hematol 2010; 85: 797-799.

3. Han J, Zhou J, SarafSL, Gordeuk VR, Calip GS. Characterization of opioid use in sickle cell disease. Pharmacoepidemiol Drug Saf 2018; 27: 479-486.

4. Levy N, Sturgess J, Mills P. "Pain as the fifth vital sign" and dependence on the "numerical pain scale" is being abandoned in the US: Why? Br J Anaesth 2018; 120: 435-438.

5. Ballas SK. The cost of health care for patients with sickle cell disease. Am J Hematol 2009; 84: 320-322.

6. World Health Organization. Saudi Arabia. Estimates of Unit Costs for Patient Services fro Saudi Arabia. [Updated 2020. Accessed 2020 January 26]. Available im URL: https://www. who.int/choice/country/sau/cost/en/

7. Ballas SK, Gupta K, Adams-Graves P. Sickle cell pain: a critical reappraisal. Blood 2012; 120: 3647-3656.

8. Carroll CP, Haywood C Jr, Fagan P, Lanzkron S. The course and correlates of high hospital utilization in sickle cell disease: Evidence from a large, urban Medicaid managed care organization. Am J Hematol 2009; 84: 666-670.

9. Shapiro, BS and Ballas, SK. The acute painful episode. in: $\mathrm{SH}$ Embury (Ed.) Sickle Cell Disease: Basic Principles and Clinical. New York (NY): Practice Raven Press; 1994. p. 531-543 
10. Martin JJ, Moore GP. Pearls, pitfalls, and updates for pain management. Emerg Med Clin North Am 1997; 15: 399-415.

11. Ahmed SG, Ibrahim UA. Prevalence of therapeutic opiate dependence among patients with sickle cell disease in Maiduguri, Nigeria. Nig J Pharm 2001; 32: 56-59.

12. Mabayoje VO, Adeyemo MA, Akinola NO. Case review: Drug addiction in sickle cell disease, a possible ongoing challenge in management of pain. J Glob Biosci 2015; 4: 2021-2025.

13. Jonassaint CR, Jones VL, Leong S, Frierson GM. A systematic review of the association between depression and health care utilization in children and adults with sickle cell disease. $\mathrm{Br} J$ Haematol 2016; 174: 136-147.

14. American Psychiatric Association. Diagnostic and Statistical Manual of Mental Disorders: DSM-5. 5th ed. Washington (DC): American Psychiatric association 2013. p. 5-25

15. Koch KL, Karafin MS, Simpson P, Field JJ. Intensive management of high-utilizing adults with sickle cell disease lowers admissions. Am J Hematol 2015; 90: 215-219.

16. Benjamin LJ, Swinson GI, Nagel RL. Sickle cell anemia day hospital: an approach for the management of uncomplicated painful crises. Blood 2000; 95: 1130-1136.

17. Wright J, Bareford D, Wright C, Augustine G, Olley K, Musamadi L, et al. Day case management of sickle pain: 3 years experience in a UK sickle cell unit. Br J Haematol 2004; 126 : 878-880.

18. Vichinsky EP. Comprehensive care in sickle cell disease: its impact on morbidity and mortality. Semin Hematol 1991; 28: 220-226.

19. Shapiro BS, Benjamin LJ, Payne R, Heidrich G. Sickle cellrelated pain: perceptions of medical practitioners. J Pain Symptom Manage 1997; 14: 168-174.

20. Pack-Mabien A, Labbe E, Herbert D, Haynes J Jr. Nurses' attitudes and practices in sickle cell pain management. Appl Nurs Res 2001; 14: 187-192.
21. Haywood C Jr, Lanzkron S, Hughes MT, Brown R, Massa $\mathrm{M}$, Ratanawongsa $\mathrm{N}$, et al. A video-intervention to improve clinician attitudes toward patients with sickle cell disease: the results of a randomized experiment. J Gen Intern Med 2011; 2 6: 518-523.

22. Mandell BF. The fifth vital sign: A complex story of politics and patient care. Cleve Clin J Med 2016; 83: 400-401.

23. Chou R, Gordon DB, de Leon-Casasola OA, Rosenberg JM, Bickler S, Brennan T, et al. Management of postoperative pain: A clinical practice guideline from the American pain society, the American society of regional anesthesia and pain medicine, and the American society of anesthesiologists' committee on regional anesthesia, executive committee, and administrative council. J Pain 2016; 17: 131-157.

24. Schug SA, Palmer GM, Scott DA, Halliwell R, Trinca J, editors. Acute Pain Management: Scientific Evidence. 4th ed. Melbourn (VIC): Australian and New Zealand College of Anaesthetists; 2015. p. 315-317.

25. Scott DA, McDONALD WM. Assessment, Measurement and History. Macintyre PE, Walker SM, Rowbotham DJ, editors. Clinical Pain Management. 2nd ed. Acute Pain. London (NW): Hodder \& Stoughton Limited. p. 135-150

26. Sinha CB, Bakshi N, Ross D, Krishnamurti L. Management of chronic pain in adults living with sickle cell disease in the era of the opioid epidemic: A qualitative study. JAMA Netw Open 2019; 2: e194410.

27. Artz N, Whelan C, Feehan S. Caring for the adult with sickle cell disease: results of a multidisciplinary pilot program. J Natl Med Assoc 2010; 102: 1009-1016. 